PROCEEDINGS OF THE

AMERICAN MATHEMATICAL SOCIETY

Volume 138, Number 5, May 2010, Pages 1821-1832

S 0002-9939(09)10134-X

Article electronically published on December 30, 2009

\title{
HAMILTONIAN STATIONARY SELF-SIMILAR SOLUTIONS FOR LAGRANGIAN MEAN CURVATURE FLOW IN THE COMPLEX EUCLIDEAN PLANE
}

\author{
ILDEFONSO CASTRO AND ANA M. LERMA
}

(Communicated by Jon G. Wolfson)

\begin{abstract}
We classify all Hamiltonian stationary Lagrangian surfaces in the complex Euclidean plane which are self-similar solutions of the mean curvature flow.
\end{abstract}

\section{INTRODUCTION}

The mean curvature flow is an evolutionary process under which a submanifold deforms in the direction of its mean curvature vector. By the first variation formula, the mean curvature vector points to the direction where the volume decreases most rapidly. Classically it has been studied by several approaches (partial differential equations, geometric measure theory, level sets or numerical methods), and its possible applications in symplectic topology and mirror symmetry are quite important.

There are very interesting results on regularity, global existence and convergence of the mean curvature flow in several ambient spaces. The flow for hypersurfaces in arbitrary Riemannian manifolds is well understood, whereas not so much is known when the codimension is greater than one. In higher codimension the mean curvature $H$ is a vector whose direction we do not know how to control, in contrast to the codimension-one case, where $H$ is essentially a scalar function whose sign is preserved along the flow. In the last few years, mean curvature flow of higher codimension submanifolds has attracted special attention, mainly when the initial submanifold is Lagrangian in complex Euclidean space $\mathbb{C}^{n}$; see [6], 7], 15], 16], [21] and 22] for example. One reason for this growing interest is that the Lagrangian condition is preserved by mean curvature flow (see [18]). In addition, as the gradient flow of the volume functional, the mean curvature flow seems to be a potential approach to the construction of special Lagrangians, which are volume minimizers that play a critical role in the $T$-duality formulation of mirror symmetry 20 .

When the ambient space is Euclidean, the mean curvature flow is the solution to a system of parabolic equations that can be considered as the heat equation for submanifolds. In general, the mean curvature flow fails to exist after a finite

Received by the editors June 17, 2009, and, in revised form, July 27, 2009.

2010 Mathematics Subject Classification. Primary 53C42, 53B25; Secondary 53D12.

Key words and phrases. Mean curvature flow, self-similar solutions, Hamiltonian stationary Lagrangian surfaces.

This research was partially supported by MEC-Feder grant MTM2007-61775.

(C)2009 American Mathematical Society Reverts to public domain 28 years from publication 
time. The singularities are completely determined by the blowup of the second fundamental form. In geometric flows, like mean curvature flow or Ricci flow, the singularities are often modelled on soliton solutions. In this article we are interested in a class of special solutions of the mean curvature flow that preserve the shape of the evolving submanifolds: the self-similar solutions, for which the evolution is a homothety. Eliminating the time variable, these self-similar solutions reduce the parabolic equation to the nonlinear elliptic equation $H=a \phi^{\perp}$, where $\phi^{\perp}$ stands for the projection of the position vector $\phi$ onto the normal space. When $a$ is a negative constant, the submanifolds shrink in finite time to a single point under the action of the mean curvature flow. If $a$ is positive, the submanifold will expand with the same shape and in this case is necessarily noncompact. If $a$ vanishes, the submanifold is minimal and stationary under the action of the flow.

By studying this type of solution we hope to give a better understanding of the flow at a singularity, since by Huisken's monotonicity formula [10, any central blowup of a finite-time singularity of the mean curvature flow is a self-similar solution.

Examples of self-similar solutions for mean curvature flow in $\mathbb{C}^{n}$ were first constructed by Anciaux [1 in 2006. In order to produce eternal solutions of the Brakke flow (a weak formulation of the mean curvature flow), Lee and Wang [12, 13] constructed in 2007 interesting examples of Hamiltonian stationary self-shrinkers and self-expanders for Lagrangian mean curvature flows, which are asymptotic to Hamiltonian stationary cones generalizing Schoen-Wolfson ones 19. All of them have been generalized very recently by Joyce, Lee and Tsui in [11] providing examples with different topologies.

It is expected that the understanding of the singularities of the mean curvature flow will rely on the classification of self-similar solutions. But it is a hard and open problem to classify all the self-similar solutions for the mean curvature flow. Using strong techniques of complex analysis, our contribution in this paper is the classification of those solutions in the complex Euclidean plane that are, in addition, Hamiltonian stationary Lagrangians, i.e. critical points of the area functional among all Hamiltonian deformations. The main result (Theorem 1) characterizes in this way three one-parameter families of examples which include Lee and Wang examples in dimension two (see Remarks 2, 3 and 5). We provide (see Propositions 2 and 3) not only Hamiltonian stationary Lagrangian conformal immersions of cylinders, Moebius strips, tori and Klein bottles but also embedded Lagrangian nontrivial planes, all of them self-similar solutions for mean curvature flow. Our construction is based essentially on a method given in [3] for obtaining Lagrangian surfaces in $\mathbb{C}^{2}$ starting from spherical and hyperbolic curves; in this setting our examples are constructed using simple particular geodesics and parallels (see Remarks 4 and 6).

\section{Hamiltonian Stationary LagRAngian SURfaces in $\mathbb{C}^{2}$}

In the complex Euclidean plane $\mathbb{C}^{2}$ we consider the bilinear Hermitian product defined by

$$
(z, w)=z_{1} \bar{w}_{1}+z_{2} \bar{w}_{2}, \quad z, w \in \mathbb{C}^{2} .
$$

Then $\langle\rangle=,\operatorname{Re}($,$) is the Euclidean metric on \mathbb{C}^{2}$ and $\omega=-\operatorname{Im}($,$) is the Kaehler$ two-form given by $\omega(\cdot, \cdot)=\langle J \cdot, \cdot\rangle$, where $J$ is the complex structure on $\mathbb{C}^{2}$. We also have that $2 \omega=d \lambda$, where $\lambda$ is known as the Liouville 1-form of $\mathbb{C}^{2}$. 
Let $\phi: M \rightarrow \mathbb{C}^{2}$ be an isometric immersion of a surface $M$ into $\mathbb{C}^{2} ; \phi$ is said to be Lagrangian if $\phi^{*} \omega=0$. Then we have $\phi^{*} T \mathbb{C}^{2}=\phi_{*} T M \oplus J \phi_{*} T M$, where $T M$ is the tangent bundle of $M$. The second fundamental form $\sigma$ of $\phi$ is given by $\sigma(v, w)=J A_{J v} w$, where $A$ is the shape operator, and so the trilinear form $C(\cdot, \cdot, \cdot)=\langle\sigma(\cdot, \cdot), J \cdot\rangle$ is fully symmetric.

Suppose $M$ is orientable and let $\omega_{M}$ be the area form of $M$. If $\Omega=d z_{1} \wedge d z_{2}$ is the closed complex-valued 2-form of $\mathbb{C}^{2}$, then $\phi^{*} \Omega=e^{i \beta} \omega_{M}$, where $\beta: M \rightarrow \mathbb{R} / 2 \pi \mathbb{Z}$ is called the Lagrangian angle map of $\phi$ (see [8]). In general, $\beta$ is a multivalued function; nevertheless $d \beta$ is a well defined closed 1-form on $M$, and its cohomology class is called the Maslov class. When $\beta$ is a single-valued function the Lagrangian is called zero-Maslov class; and if $\cos \beta \geq \epsilon$ for some $\epsilon>0$, then the Lagrangian is said to be almost calibrated.

Note that the Lagrangian condition implies that the Liouville 1-form $\lambda$ is a closed 1 -form on $M$. A Lagrangian submanifold is said to be monotone if $[\lambda]=c[d \beta]$, for some positive constant $c$. The standard examples of monotone Lagrangians in $\mathbb{C}^{2}$ are the Clifford tori

$$
T_{r} \equiv\left\{\left(z_{1}, z_{2}\right) \in \mathbb{C}^{2}:\left|z_{1}\right|=\left|z_{2}\right|=r\right\}, r>0 .
$$

It is remarkable that $\beta$ satisfies (see for example [19])

$$
J \nabla \beta=2 H,
$$

where $H$ is the mean curvature vector of $\phi$, defined by $H=(1 / 2) \operatorname{trace} \sigma$. (We point out that some other authors, e.g. [12, [13, consider $H=\operatorname{trace} \sigma$ and then $J \nabla \beta=H$.)

If $\beta$ is constant, say $\beta \equiv \beta_{0}$ (or equivalently $H=0$ ), then the Lagrangian immersion $\phi$ is calibrated by $\operatorname{Re}\left(e^{-i \beta_{0}} \Omega\right)$ and hence area-minimizing. They are referred to as being Special Lagrangian.

A Lagrangian submanifold is called Hamiltonian stationary if the Lagrangian angle $\beta$ is harmonic; i.e. $\Delta \beta=0$, where $\Delta$ is the Laplace operator on $M$. Hamiltonian stationary Lagrangian (in short HSL) surfaces are critical points of the area functional with respect to a special class of infinitesimal variations preserving the Lagrangian constraint, namely, the class of compactly supported Hamiltonian vector fields (see [17). Examples of HSL surfaces in $\mathbb{C}^{2}$ can be found in [2], 5] and [9].

\section{Self-Similar solutions for the MeAn CURVAture Flow}

Let $\phi: M \rightarrow \mathbb{R}^{4}$ be an immersion of a smooth surface $M$ in Euclidean 4-space. It is not hard to check that

$$
2 H=\Delta \phi
$$

where $H$ is the mean curvature vector and $\Delta$ is the Laplace operator of the induced metric on $M$.

The mean curvature flow (in short MCF) of $\phi: M \rightarrow \mathbb{R}^{4}$ is a family of immersions $F: M \times\left[0, T_{\text {sing }}\right) \rightarrow \mathbb{R}^{4}$ parameterized by $t$ that satisfies

$$
\frac{d}{d t} F_{t}(p)=H(p, t), \quad F_{0}=\phi,
$$

where $H(p, t)$ is the mean curvature vector of $F_{t}(M)$ at $F_{t}(p)=F(p, t)$ and $\left[0, T_{\text {sing }}\right)$ is the maximal time interval such that (3) holds. Looking at equation (2), this can be considered as the heat equation for submanifolds. 
The equation (3) is a quasi-linear parabolic system, and short time existence is guaranteed when the initial submanifold $M$ is compact.

When $\phi: M \rightarrow \mathbb{R}^{4} \equiv \mathbb{C}^{2}$ is Lagrangian it is well known that $0<T_{\text {sing }}<\infty$, and being Lagrangian it is preserved along the mean curvature flow. The monotone condition defined in Section 2 is also preserved under the flow (see [7]).

In geometric flows such as the Ricci flow or the MCF, singularities are often locally modelled on soliton solutions. In the case of MCF, one type of soliton solutions of great interest are those moved by scaling in the Euclidean space. We recall that solitons moved by scaling must be of the following form:

Definition 1. An immersion $\phi: M \rightarrow \mathbb{R}^{4}$ is called a self-similar solution for mean curvature flow if

$$
H=a \phi^{\perp}
$$

for some nonzero constant $a$, where $\phi^{\perp}$ denotes the normal projection of the position vector $\phi$ and $H$ is the mean curvature vector of $\phi$. If $a<0$ it is called a self-shrinker, and if $a>0$ it is called a self-expander.

Remark 1. In the Lagrangian setting, using (1), we can reformulate condition (4) in terms of the Lagrangian angle map and the restriction to the surface of the Liouville 1-form by means of

$$
d \beta=-4 a \phi^{*} \lambda, a \neq 0 .
$$

In particular, any Lagrangian self-similar solution for MCF is monotone.

If $\phi$ is a self-similar solution, then $F_{t}:=\sqrt{2 a t+1} \phi, 2 a t+1>0$, is a solution of (3). When $a=0$ the submanifold is minimal, i.e. $H=0$, and is fixed by MCF.

One could normalize the value of $a$ up to dilations, but we prefer not to do it because we will take different particular values of $a$ along the paper to recover some known examples.

It is an exercise to check that, given any $a<0$, the right circular cylinder $\mathbb{S}^{1}\left(\frac{1}{\sqrt{-2 a}}\right) \times \mathbb{R}$ and the Clifford torus $\mathbb{S}^{1}\left(\frac{1}{\sqrt{-2 a}}\right) \times \mathbb{S}^{1}\left(\frac{1}{\sqrt{-2 a}}\right)$ satisfy equation (44). Thus they are examples of self-shrinkers. Both of them are also Lagrangian surfaces with parallel mean curvature vector and hence Hamiltonian stationary Lagrangians.

We finish this section by studying a close relationship between the area and the Willmore functional of compact Lagrangian self-shrinkers that is even true for monotone compact HSL surfaces; see [14.

Proposition 1. Let $\phi: M \rightarrow \mathbb{C}^{2}$ be a Lagrangian self-similar solution for mean curvature flow, i.e. $H=a \phi^{\perp}, a \neq 0$. Then

$$
\operatorname{div} \phi^{\top}=2\left(1+\frac{|H|^{2}}{a}\right)
$$

where $\phi^{\top}$ denotes the tangent projection of the position vector $\phi$. In particular, if $M$ is compact, then $a<0$ and $\operatorname{Area}(M)=-\frac{1}{a} \int_{M}|H|^{2}$.

Proof. Derivating in the direction of any tangent vector $v$ in the equality $\phi=$ $\phi^{\top}+H / a$ and taking tangent components, we get that $v=\nabla_{v} \phi^{\top}-\frac{1}{a} A_{H} v$. If $\left\{e_{1}, e_{2}\right\}$ is an orthonormal frame in $M$, then $\operatorname{div} \phi^{\top}=2+\frac{1}{a} \sum_{i=1}^{2}\left\langle A_{H} e_{i}, e_{i}\right\rangle=2+\frac{2}{a}|H|^{2}$. 


\section{The EXAMPles}

In this section we introduce three one-parameter families of HSL surfaces in $\mathbb{C}^{2}$ which are self-similar solutions for mean curvature flow satisfying (4) and describe their main geometric properties. In the next result, we provide examples with the topology of a plane, a cylinder or a Moebius strip.

Proposition 2. Given any $a>0$, let us define

$$
\begin{aligned}
& \Phi_{\delta}: \mathbb{R}^{2} \rightarrow \mathbb{C}^{2}, \delta>0, \\
& \Phi_{\delta}(s, t)=\frac{1}{\sqrt{2 a}}\left(i \mathrm{~s}_{\delta} \cosh t e^{-\frac{i s}{c_{\delta}}}, \mathrm{t}_{\delta} \sinh t e^{i \mathrm{c}_{\delta} s}\right),
\end{aligned}
$$

with $\mathrm{s}_{\delta}=\sinh \delta, \mathrm{c}_{\delta}=\cosh \delta$ and $\mathrm{t}_{\delta}=\tanh \delta$. Then $\Phi_{\delta}$ is a Hamiltonian stationary Lagrangian conformal immersion and a self-similar solution for mean curvature flow satisfying (4).

If $\cosh ^{2} \delta \notin \mathbb{Q}, \Phi_{\delta}$ is, in addition, an embedded self-expander plane asymptotic to the Hamiltonian stationary Lagrangian cone

$$
\left\{\left(i x_{1} e^{-\frac{i s}{c_{\delta}}}, x_{2} e^{i \mathrm{c}_{\delta} s}\right):\left(x_{1}, x_{2}\right) \in \mathbb{R}^{2}, s \in \mathbb{R}, x_{1}^{2}=\cosh ^{2} \delta x_{2}^{2}\right\} .
$$

If $\cosh ^{2} \delta=p / q \in \mathbb{Q},(p, q)=1, p>q$, then $\Phi_{\delta}$ is given by

$$
\begin{aligned}
& \Phi_{p, q}: \mathbb{R}^{2} \rightarrow \mathbb{C}^{2}, p>q, \\
& \Phi_{p, q}(s, t)=\sqrt{\frac{p-q}{2 a}}\left(\frac{i}{\sqrt{q}} \cosh t e^{-i \sqrt{\frac{q}{p}} s}, \frac{1}{\sqrt{p}} \sinh t e^{i \sqrt{\frac{p}{q}} s}\right)
\end{aligned}
$$

and satisfies $\Phi_{p, q}(s+2 \pi \sqrt{p q}, t)=\Phi_{p, q}(s, t), \forall(s, t) \in \mathbb{R}^{2}$, inducing a Hamiltonian stationary Lagrangian immersion of a self-expander cylinder.

Moreover, if $p$ is odd and $q$ is even, then $\Phi_{p, q}(s+\pi \sqrt{p q},-t)=\Phi_{p, q}(s, t), \forall(s, t) \in$ $\mathbb{R}^{2}$, inducing a Hamiltonian stationary Lagrangian immersion of a self-expander Moebius strip.

Given any $a<0$, let us define

$$
\begin{aligned}
& \Upsilon_{\gamma}: \mathbb{R}^{2} \rightarrow \mathbb{C}^{2}, 0<\gamma<\pi / 2, \\
& \Upsilon_{\gamma}(s, t)=\frac{1}{\sqrt{-2 a}}\left(-i \mathrm{~s}_{\gamma} \cosh t e^{\frac{i s}{c_{\gamma}}}, \mathrm{t}_{\gamma} \sinh t e^{-i c_{\gamma} s}\right),
\end{aligned}
$$

with $\mathrm{s}_{\gamma}=\sin \gamma, \mathrm{c}_{\gamma}=\cos \gamma$ and $\mathrm{t}_{\gamma}=\tan \gamma$. Then $\Upsilon_{\gamma}$ is a Hamiltonian stationary Lagrangian conformal immersion and a self-similar solution for mean curvature flow satisfying (4).

If $\cos ^{2} \gamma \notin \mathbb{Q}, \Upsilon_{\gamma}$ is, in addition, an embedded self-shrinker plane asymptotic to the Hamiltonian stationary Lagrangian cone

$$
\left\{\left(-i x_{1} e^{\frac{i s}{c_{\gamma}}}, x_{2} e^{-i \mathrm{c}_{\gamma} s}\right):\left(x_{1}, x_{2}\right) \in \mathbb{R}^{2}, s \in \mathbb{R}, x_{1}^{2}=\cos ^{2} \gamma x_{2}^{2}\right\} .
$$

If $\cos ^{2} \gamma=p / q \in \mathbb{Q},(p, q)=1, p<q$, then $\Upsilon_{\gamma}$ is given by

$$
\begin{aligned}
& \Upsilon_{p, q}: \mathbb{R}^{2} \rightarrow \mathbb{C}^{2}, p<q, \\
& \Upsilon_{p, q}(s, t)=\sqrt{\frac{q-p}{-2 a}}\left(\frac{-i}{\sqrt{q}} \cosh t e^{i \sqrt{\frac{q}{p}} s}, \frac{1}{\sqrt{p}} \sinh t e^{-i \sqrt{\frac{p}{q}} s}\right)
\end{aligned}
$$

and satisfies $\Upsilon_{p, q}(s+2 \pi \sqrt{p q}, t)=\Upsilon_{p, q}(s, t), \forall(s, t) \in \mathbb{R}^{2}$, inducing a Hamiltonian stationary Lagrangian immersion of a self-shrinker cylinder. 
Moreover, if $q$ is even and $p$ is odd, then $\Upsilon_{p, q}(s+\pi \sqrt{p q},-t)=\Upsilon_{p, q}(s, t), \forall(s, t) \in$ $\mathbb{R}^{2}$, inducing a Hamiltonian stationary Lagrangian immersion of a self-shrinker Moebius strip.

Remark 2. We can recover the Hamiltonian stationary expanders $\mathcal{E}$ and the Hamiltonian stationary shrinkers $\mathcal{S}$ studied in Proposition 2.1 of [12] by Lee and Wang, taking $\mathcal{E}(u, \theta)=\Phi_{p, q}(\sqrt{p q} \theta, u)$ and $\mathcal{S}(u, \theta)=i \Upsilon_{p, q}(\sqrt{p q} \theta, u)$ with $\Phi_{p, q}$ given respectively in (6) and $\Upsilon_{p, q}$ in (8), $a$ being equal to $\frac{p-q}{2 p q}$.

Remark 3. By taking $x_{1}=\sqrt{\frac{p-q}{2 a}} \frac{\cosh t}{\sqrt{q}}>0$ and $x_{2}=\sqrt{\frac{p-q}{2 a}} \frac{\sinh t}{\sqrt{p}}$ we can rewrite

$$
\left(\begin{array}{cc}
-i & 0 \\
0 & 1
\end{array}\right) \Phi_{p, q}(\sqrt{p q} \theta, t)=\left(x_{1} e^{-i q \theta}, x_{2} e^{i p \theta}\right),-q x_{1}^{2}+p x_{2}^{2}=\frac{q-p}{2 a}<0
$$

and

$$
\left(\begin{array}{cc}
i & 0 \\
0 & 1
\end{array}\right) \Upsilon_{p, q}(\sqrt{p q} \theta, t)=\left(x_{1} e^{i q \theta}, x_{2} e^{-i p \theta}\right), q x_{1}^{2}-p x_{2}^{2}=\frac{q-p}{-2 a}>0,
$$

where $0 \leq \theta<2 \pi$. In this way we arrive at the examples given in Proposition 2.1 of [13] for $n=2$. Restricting $\theta \in[0, \pi)$, in Proposition 2.2 of [13] it is proved that these examples are oriented if and only if $p-q$ is even and are embedded if and only if $q=1$.

We finally remark that $\Phi_{\delta}$ and $\Upsilon_{\gamma}$ generalize them, although they can be included in the observation made in Remark 2.1 of [13].

Remark 4. Following the spirit of [3], the Lagrangians $\Phi_{\delta}$ (resp. $\Upsilon_{\gamma}$ ) are constructed with the Legendrian geodesic $t \rightarrow(\sinh t, \cosh t)$ in the anti-de Sitter space $\mathbb{H}_{3}^{1}$ and the constant curvature Legendrian curves $s \rightarrow \frac{1}{\sqrt{2 a}}\left(\mathrm{t}_{\delta} e^{i \mathrm{c}_{\delta} s}, i \mathrm{~s}_{\delta} e^{-i s / \mathrm{c}_{\delta}}\right.$ ) (resp. $\left.s \rightarrow \frac{1}{\sqrt{2 a}}\left(\mathrm{t}_{\gamma} e^{-i \mathrm{c}_{\gamma} s},-i \mathrm{~s}_{\gamma} e^{i s / \mathrm{c}_{\gamma}}\right)\right)$ in 3-spheres.

Proof. Given any $a>0$, let $\Phi=\Phi_{\delta}$. It is an exercise to check that

$$
\left|\Phi_{s}\right|^{2}=\frac{1}{2 a}\left(\mathrm{t}_{\delta}^{2} \cosh ^{2} t+\mathrm{s}_{\delta}^{2} \sinh ^{2} t\right)=\left|\Phi_{t}\right|^{2},\left(\Phi_{s}, \Phi_{t}\right)=0 .
$$

This shows that $\Phi$ is a Lagrangian conformal immersion whose induced metric is given by $\langle\rangle=,e^{2 u(t)}\left(d s^{2}+d t^{2}\right)$, with $e^{2 u(t)}=\frac{1}{2 a}\left(\mathrm{t}_{\delta}^{2} \cosh ^{2} t+\mathrm{s}_{\delta}^{2} \sinh ^{2} t\right)$. Moreover we compute $e^{i \beta_{\Phi}}=e^{-2 u} \operatorname{det}_{\mathbb{C}}\left(\Phi_{s}, \Phi_{t}\right)=e^{i \mathrm{~s}_{\delta}^{2} s / \mathrm{c}_{\delta}}$. So we conclude that $\Phi$ is HSL since $\beta_{\Phi}$ depends only on $s$. Using (11) it is not hard to obtain that the mean curvature vector of $\Phi$ satisfies that $H_{\Phi}=\frac{\mathrm{s}_{\delta}^{2} e^{-2 u}}{2 \mathrm{c}_{\delta}} J \Phi_{s}=a \Phi^{\perp}$.

To prove that $\Phi$ is an embedding, we start from $\Phi\left(s_{1}, t_{1}\right)=\Phi\left(s_{2}, t_{2}\right)$, and then it is clear that when $\left(s_{1}, t_{1}\right) \neq\left(s_{2}, t_{2}\right), \mathrm{c}_{\delta}^{2}$ is necessarily a rational number.

Finally, $\Phi$ is asymptotic to the given HSL cone, taking into account that if $t \rightarrow+\infty$, then $\cosh t, \sinh t \simeq e^{t} / 2$ and if $t \rightarrow-\infty$, then $\cosh t \simeq e^{-t} / 2$ and $\sinh t \simeq-e^{-t} / 2$.

The reasoning for $\Upsilon_{\gamma}$ is completely similar, so we omit it.

The next result describes examples with the topology of a cylinder, a torus or a Klein bottle. 
Proposition 3. Given any $a<0$, let us define

$$
\begin{aligned}
& \Psi_{\nu}: \mathbb{S}^{1} \times \mathbb{R} \rightarrow \mathbb{C}^{2}, \nu>0, \\
& \Psi_{\nu}\left(e^{i s}, t\right)=\frac{1}{\sqrt{-2 a}}\left(\mathrm{c}_{\nu} \cos s e^{\frac{i t}{\mathrm{~s}_{\nu}}}, \mathrm{t}_{\nu} \sin s e^{i \mathrm{~s}_{\nu} t}\right),
\end{aligned}
$$

with $\mathrm{s}_{\nu}=\sinh \nu, \mathrm{c}_{\nu}=\cosh \nu$ and $\mathrm{t}_{\nu}=\operatorname{coth} \nu$. Then $\Psi_{\nu}$ is a Hamiltonian stationary Lagrangian conformal immersion and a self-similar solution for mean curvature flow satisfying (4). If $\sinh ^{2} \nu \notin \mathbb{Q}, \Psi_{\nu}$ is, in addition, an embedded self-shrinker cylinder.

If $\sinh ^{2} \nu=m / n \in \mathbb{Q},(m, n)=1$, then $\Psi_{\nu}$ is given by

$$
\begin{aligned}
& \Psi_{m, n}: \mathbb{S}^{1} \times \mathbb{R} \rightarrow \mathbb{C}^{2},(m, n)=1, \\
& \Psi_{m, n}(s, t)=\sqrt{\frac{m+n}{-2 a}}\left(\frac{1}{\sqrt{n}} \cos s e^{i \sqrt{\frac{n}{m}} t}, \frac{1}{\sqrt{m}} \sin s e^{i \sqrt{\frac{m}{n}} t}\right) .
\end{aligned}
$$

Moreover, $\Psi \equiv \Psi_{m, n}$ satisfies the following properties:

(1) $\Psi(s+2 \pi, t)=\Psi(s, t)=\Psi(s, t+2 \pi \sqrt{m n}), \forall(s, t) \in \mathbb{R}^{2}$; if, in addition, $m$ and $n$ are odd, then $\Psi(s+\pi, t+\pi \sqrt{m n})=\Psi(s, t), \forall(s, t) \in \mathbb{R}^{2}$. Let $\Lambda_{m, n}$ be the lattice spanned by $(2 \pi, 0)$ and $(0,2 \pi \sqrt{m n})$ (resp. $(2 \pi, 0)$ and $(\pi, \pi \sqrt{m n})$ ) when $m$ or $n$ are even (resp. when $m$ and $n$ are odd) and $\mathcal{T}_{m, n}=\mathbb{R}^{2} / \Lambda_{m, n}$ the corresponding self-shrinker torus. Then:

$$
\operatorname{Area}\left(\mathcal{T}_{m, n}\right)= \begin{cases}\frac{(m+n)^{2} \pi^{2}}{-a \sqrt{m n}}, & m \text { or } n \text { even, } \\ \frac{(m+n)^{2} \pi^{2}}{-2 a \sqrt{m n}}, & m \text { and } n \text { odd. }\end{cases}
$$

(2) If $m$ is odd and $n$ is even, then $\Psi(2 \pi-s, t+\pi \sqrt{m n})=\Psi(s, t), \forall(s, t) \in \mathbb{R}^{2}$. If $m$ is even and $n$ is odd, then $\Psi(\pi-s, t+\pi \sqrt{m n})=\Psi(s, t), \forall(s, t) \in \mathbb{R}^{2}$. In both cases, $m+n$ is odd and $\mathcal{T}_{m, n}$ is the covering of the corresponding self-shrinker Klein bottle $\mathcal{K}_{m, n}$.

(3) The Clifford torus $\mathcal{T}_{1,1}$ is the only one embedded in the above family.

Remark 5. The immersion $\Psi_{\operatorname{arcsinh} 1} \equiv \Psi_{1,1}$ corresponding to the Clifford torus $T_{1 / \sqrt{-2 a}}, a<0$, can be checked to be the only Willmore surface in this family. Up to isometries, it is enough to consider $\nu \in(0, \operatorname{arcsinh} 1$ ] (and hence $m \leq n)$ because $\Psi_{\hat{\nu}}$, with $\hat{\nu}=\log (\operatorname{coth} \nu / 2)$, is congruent to $\Psi_{\nu}$.

By taking $x_{1}=\sqrt{\frac{m+n}{-2 a}} \frac{\cos s}{\sqrt{n}}$ and $x_{2}=\sqrt{\frac{m+n}{-2 a}} \frac{\sin s}{\sqrt{m}}$, we can rewrite

$$
\Psi_{m, n}(s, \sqrt{m n} \theta)=\left(x_{1} e^{i n \theta}, x_{2} e^{i m \theta}\right), n x_{1}^{2}+m x_{2}^{2}=C=\frac{m+n}{-2 a}>0 .
$$

Therefore we arrive again at other examples considered in Proposition 2.1 of 13 . by Lee and Wang when $n=2$. We remark that $\Psi_{\nu}$ generalizes them, although they can be included in the observation made in Remark 2.1 of [13].

Using Proposition 1 we also get that the Willmore functional $\mathcal{W}:=\int_{M}|H|^{2}$ of the tori $\mathcal{T}_{m, n}$ is given by

$$
\mathcal{W}\left(\mathcal{T}_{m, n}\right)= \begin{cases}\frac{(m+n)^{2} \pi^{2}}{\sqrt{m n}}, & m \text { or } n \text { even, } \\ \frac{(m+n)^{2} \pi^{2}}{2 \sqrt{m n}}, & m \text { and } n \text { odd. }\end{cases}
$$


Remark 6. Following the spirit of [3], the Lagrangians $\Psi_{\nu}$ are constructed with the constant curvature Legendrian curves $t \rightarrow \frac{1}{\sqrt{-2 a}}\left(\mathrm{c}_{\nu} e^{i t / \mathrm{s}_{\nu}}, \mathrm{t}_{\nu} e^{i \mathrm{~s}_{\nu} t}\right)$ in anti-de Sitter spaces and the Legendrian geodesic $s \rightarrow(\cos s, \sin s)$ in the unit 3-sphere.

On the other hand, it is clear that the HSL tori $\mathcal{T}_{m, n}$ admit a one-parameter group of isometries. Using the notation of [5], it is not complicated to check that their universal coverings would correspond to the immersions $\mathcal{F}_{0, \beta}^{\pi / 2}, \sin \beta \in \mathbb{Q}$.

Proof. Given any $a<0$, let $\Psi=\Psi_{\nu}$. It is an exercise to check that

$$
\left|\Psi_{s}\right|^{2}=\frac{1}{-2 a}\left(\mathrm{t}_{\nu}^{2} \cos ^{2} s+\mathrm{c}_{\nu}^{2} \sin ^{2} s\right)=\left|\Psi_{t}\right|^{2},\left(\Psi_{s}, \Psi_{t}\right)=0 .
$$

This shows that $\Psi$ is a Lagrangian conformal immersion whose induced metric is given by $\langle\rangle=,e^{2 v(s)}\left(d s^{2}+d t^{2}\right)$, with $e^{2 v(s)}=\frac{1}{-2 a}\left(\mathrm{t}_{\nu}^{2} \cos ^{2} s+\mathrm{c}_{\nu}^{2} \sin ^{2} s\right)$. Moreover we get that $e^{i \beta_{\Psi}(t)}=-i e^{i \mathrm{c}_{\nu}^{2} t / \mathrm{s}_{\nu}}$. Thus we conclude that $\Psi$ is HSL. Using (11) it is easy to obtain that the mean curvature vector of $\Psi$ satisfies $H_{\Psi}=\frac{\mathrm{c}_{\nu}^{2} e^{-2 v}}{2 \mathrm{~s}_{\nu}} J \Psi_{t}=$ $a \Psi^{\perp}$.

It is straightforward to get that $\Psi$ is an embedding since $\Psi\left(s_{1}, t_{1}\right)=\Psi\left(s_{2}, t_{2}\right)$ and $\left(s_{1}, t_{1}\right) \neq\left(s_{2}, t_{2}\right)$ imply that $\mathrm{s}_{\nu}^{2}$ is a rational number.

The geometric properties of $\Psi_{m, n}$ are deduced by making use of the above data and Remark 4.

\section{Classification}

Theorem 1. Let $\phi: M^{2} \rightarrow \mathbb{C}^{2}$ be a Hamiltonian stationary Lagrangian self-similar solution for mean curvature flow.

(a) If $\phi$ is a self-expander, i.e. $H=a \phi^{\perp}, a>0$, then $\phi$ is locally congruent to some $\Phi_{\delta}: \mathbb{R}^{2} \rightarrow \mathbb{C}^{2}, \delta>0$, described in Proposition 2 .

(b) If $\phi$ is a self-shrinker, i.e. $H=a \phi^{\perp}, a<0$, then $\phi$ is locally congruent to some of the following:

(i) the right circular cylinder $\mathbb{S}^{1}\left(\frac{1}{\sqrt{-2 a}}\right) \times \mathbb{R}$;

(ii) the Clifford torus $\mathbb{S}^{1}\left(\frac{1}{\sqrt{-2 a}}\right) \times \mathbb{S}^{1}\left(\frac{1}{\sqrt{-2 a}}\right)$;

(iii) some $\Upsilon_{\gamma}: \mathbb{R}^{2} \rightarrow \mathbb{C}^{2}, 0<\gamma<\pi / 2$, described in Proposition 2 ,

(iv) some $\Psi_{\nu}: \mathbb{S}^{1} \times \mathbb{R} \rightarrow \mathbb{C}^{2}, \nu>0$, described in Proposition 3 ,

Proof. First, following [4, we can associate to any Lagrangian immersion $\phi: M \longrightarrow$ $\mathbb{C}^{2}$ a cubic differential form $\Theta$ on $M$ defined by

$$
\Theta(z)=f(z)(d z)^{3}, \text { with } f(z)=4 C\left(\partial_{z}, \partial_{z}, \partial_{z}\right)
$$

and a differential form $\Lambda$ on $M$ defined by

$$
\Lambda(z)=\bar{h}(z) d z, \text { with } h(z)=2 \omega\left(\partial_{\bar{z}}, H\right),
$$

where $z=x+i y$ is a local isothermal coordinate on $M$ such that the induced metric, also denoted by $\langle$,$\rangle , is written as \langle\rangle=,e^{2 u}|d z|^{2}$ with $|d z|^{2}$ the Euclidean metric, and where $C$ and $\omega$ are extended $\mathbb{C}$-linearly to the complexified tangent bundles. We remark that our $h$ here is $\bar{h}$ in [4].

It is straightforward to check that the Frenet equations of $\phi$ are given by

$$
\begin{array}{r}
\phi_{z z}=2 u_{z} \phi_{z}+\frac{\bar{h}}{2} J \phi_{z}+\frac{e^{-2 u} f}{2} J \phi_{\bar{z}}, \\
\phi_{z \bar{z}}=\frac{h}{2} J \phi_{z}+\frac{\bar{h}}{2} J \phi_{\bar{z}},
\end{array}
$$


and it is not difficult (see equation (3.3) in [4]) to get the compatibility equations for (11), obtaining

$$
\begin{array}{r}
4 u_{z \bar{z}}+\frac{|h|^{2}-e^{-4 u}|f|^{2}}{2}=0, \\
\operatorname{Im}\left(h_{z}\right)=0, \\
\overline{f_{\bar{z}}}=e^{2 u}\left(h_{\bar{z}}-2 u_{\bar{z}} h\right) .
\end{array}
$$

Now (1) translates into $h=\beta_{\bar{z}}$.

Since $\phi$ is a self-similar solution for mean curvature flow, using (4) we have that $h=-2 a\left\langle\phi_{\bar{z}}, J \phi\right\rangle, a \neq 0$, and from (11) we deduce that

$$
h_{z}=-a \operatorname{Re}\left(h|\phi|_{z}^{2}\right) .
$$

Taking into account that $\left\langle\phi_{z}, \phi_{\bar{z}}\right\rangle=e^{2 u} / 2$ and $\left\langle\phi_{z}, \phi_{z}\right\rangle=0$, from (11) we also have that

$$
|\phi|_{z \bar{z}}^{2}=\frac{|h|^{2}}{a}+e^{2 u}
$$

and

$$
|\phi|_{z z}^{2}=2 u_{z}|\phi|_{z}^{2}+\frac{1}{2 a}\left(\bar{h}^{2}+e^{-2 u} f h\right) .
$$

As $\phi$ is also a Hamiltonian stationary Lagrangian immersion, the second equation of (12) implies that $\bar{h}_{\bar{z}}=h_{z}=\beta_{z \bar{z}}=0$. Hence $\Lambda$ is a holomorphic differential, and we can normalize $h \equiv \mu, \mu>0$, since the zeroes of $h$ and $H$ are the same and $a \neq 0$. Thus (13) says that $g:=|\phi|^{2}$ satisfies $g_{x}=0$; that is, $g=g(y)$. In addition, from (14), $g$ satisfies

$$
g^{\prime \prime}=4\left(\frac{\mu^{2}}{a}+e^{2 u}\right) .
$$

In particular, we get that $u=u(y)$ too. We can now express $f$ in terms of $g$ and $u$ from (15) by

$$
f=\frac{e^{2 u}}{\mu}\left(a u^{\prime} g^{\prime}-\frac{a}{2} g^{\prime \prime}-\mu^{2}\right) .
$$

If $\phi^{\top}$ denotes the tangent part of $\phi$, using $|h|^{2}=e^{2 u}|H|^{2}$ and taking modules in the equality $\phi=\phi^{\top}+H / a$, we get

$$
g=e^{-2 u}\left(\frac{g^{\prime 2}}{4}+\frac{\mu^{2}}{a^{2}}\right) .
$$

This implies that $g>0$.

From (16) and (18) we arrive at the following o.d.e. for $g$ :

$$
a^{2}\left(g g^{\prime \prime}-g^{\prime 2}\right)=4 \mu^{2}(1+a g) .
$$

Only when $a<0$ does the equation (19) have a constant solution $g \equiv-1 / a$. In this case (16) or (18) gives $e^{2 u} \equiv-\mu^{2} / a$ and (17) gives $f \equiv \mu^{3} / a$. The integration of the corresponding Frenet equations (11), now simply written as $\phi_{x x}=\phi_{y y}=$ $\mu J \phi_{x}, \phi_{x y}=\mu J \phi_{y}$, leads to the Clifford torus $\mathbb{S}^{1}\left(\frac{1}{\sqrt{-2 a}}\right) \times \mathbb{S}^{1}\left(\frac{1}{\sqrt{-2 a}}\right)$. This proves part (b)(ii).

In the general case, we obtain a first integral for (19) given by

$$
g^{\prime 2}=P(g):=4 E g^{2}-\frac{8 \mu^{2}}{a} g-\frac{4 \mu^{2}}{a^{2}}, \quad E \in \mathbb{R} .
$$


We now look for the o.d.e. for the conformal factor of the induced metric. Using (18) and (20) we have that

$$
e^{2 u}=E g-2 \mu^{2} / a
$$

Then (20) translates into

$$
u^{\prime 2}-\frac{2 \mu^{2} E}{a} e^{-2 u}+\frac{\mu^{2} E^{2}}{a^{2}} e^{-4 u}=E,
$$

and so

$$
u^{\prime \prime}+\frac{2 \mu^{2} E}{a} e^{-2 u}-\frac{2 \mu^{2} E^{2}}{a^{2}} e^{-4 u}=0 .
$$

Using (21) and (22), (17) implies that

$$
f=\mu\left(e^{2 u}-2 E / a\right)
$$

which is compatible with (12) and (23).

Then we can rewrite the Frenet equations (11) in the following way:

$$
\begin{aligned}
\phi_{x x} & =-u^{\prime} \phi_{y}+\left(2 \mu-\frac{\mu E}{a} e^{-2 u}\right) J \phi_{x}, \\
\phi_{x y} & =u^{\prime} \phi_{x}+\frac{\mu E}{a} e^{-2 u} J \phi_{y}, \\
\phi_{y y} & =u^{\prime} \phi_{y}+\frac{\mu E}{a} e^{-2 u} J \phi_{x} .
\end{aligned}
$$

After a long computation, using (25), (22) and (23), we get that $\phi_{x y y}=E \phi_{x}$ and $\phi_{y y y}=E \phi_{y}$. Up to translations, we can consider

$$
\phi_{y y}=E \phi,
$$

and (25) gives

$$
\phi_{x x}=-E \phi+2 \mu J \phi_{x}
$$

In particular, $H=\mu e^{-2 u} J \phi_{x}$.

On the one hand, when $E=0$ it follows necessarily that $a<0$ from (20). Using (21), we obtain that $e^{2 u} \equiv-2 \mu^{2} / a$ and (24) gives $f \equiv-2 \mu^{3} / a$. The integration of the corresponding Frenet equations (25), now simply written as $\phi_{x x}=2 \mu J \phi_{x}, \phi_{x y}=\phi_{y y}=0$, leads to the right circular cylinder $\mathbb{S}^{1}\left(\frac{1}{\sqrt{-2 a}}\right) \times \mathbb{R}$. This proves part (b)(i).

On the other hand, when $E \neq 0$, the discriminant of the second order polynomial $P(g)$ in (20) is $64 \mu^{2}\left(\mu^{2}+E\right) / a^{2}$. As $P(g)=g^{\prime 2}$ must be nonnegative for $g>0$ (and observe that $P(0)<0$ ) we distinguish two cases according to the sign of the energy $E$ to reach the following conclusions:

- Case (a): If $E>0$, then $g$ is bounded from below.

- Case (b): If $E<0$, we necessarily have $E \geq-\mu^{2}$, and $g$ is bounded from below and from above. We remark that (21) shows that if $E<0$, then necessarily $a<0$, and we also point out that the case $E=-\mu^{2}$ corresponds precisely with the constant case $g \equiv 1 /-a$.

We now proceed to integrate explicitly (25) through (26) and (27) . From (21) there is no restriction supposing $u^{\prime}(0)=0$. Let $\alpha:=e^{2 u(0)}>0$. So (22) says that

$$
E=a \alpha\left(2+\frac{a \alpha}{\mu^{2}}\right) \text {. }
$$


Case (a) $(E>0)$. Using (25), (26) and (27), we get

$$
\phi(x, y)=\cosh (\sqrt{E} y) C_{1}(x)+\sinh (\sqrt{E} y) C_{2}(x),
$$

where $C_{1}(x)=\frac{\mu}{a \alpha} J \phi_{x}(x, 0)$ and $C_{2}(x)=\frac{1}{\sqrt{E}} \phi_{y}(x, 0)$. It is clear that $\left(C_{1}(x), C_{2}(x)\right)$ $=0$. In addition, (25) and (28) imply that $C_{1}^{\prime}(x)=\frac{-i a \alpha}{\mu} C_{1}(x)$ and $C_{2}^{\prime}(x)=$ $\frac{i \mu E}{a \alpha} C_{2}(x)$.

Choosing in $\mathbb{C}^{2}$ the unitary reference $\varepsilon_{1}=\phi_{x}(0,0) / \sqrt{\alpha}, \varepsilon_{2}=\phi_{y}(0,0) / \sqrt{\alpha}$, we arrive at

$$
\phi(x, y)=\left(\frac{i \mu}{a \sqrt{\alpha}} \cosh (\sqrt{E} y) \exp \left(-\frac{i a \alpha}{\mu} x\right), \frac{\sqrt{\alpha}}{\sqrt{E}} \sinh (\sqrt{E} y) \exp \left(\frac{i \mu E}{a \alpha} x\right)\right) .
$$

Introducing the new parameter $b:=g(0)>0$ and using (18), we have $b=\frac{\mu^{2}}{\alpha a^{2}}$ and (28) gives that $1+2 a b=\frac{\mu^{2} E}{\alpha^{2} a^{2}}$. We observe that if $a<0$, then $0<b<-1 /(2 a)$ in order to get $E>0$. Changing coordinates with $x+i y=\frac{1}{\sqrt{E}}(s+i t)$ we finally get

$$
\phi(s, t)=\left( \pm i \sqrt{b} \cosh t \exp \left(\frac{\mp i s}{\sqrt{1+2 a b}}\right), \frac{\sqrt{b}}{\sqrt{1+2 a b}} \sinh t \exp ( \pm i \sqrt{1+2 a b} s)\right),
$$

using the sign \pm according to $a \gtrless 0$. If $a>0$, we put $b=\frac{\sinh ^{2} \delta}{2 a}, \delta>0$, and this proves part (a); if $a<0$, we put $b=\frac{\sin ^{2} \gamma}{-2 a}, 0<\gamma<\pi / 2$, and this proves part (b)(iii).

Case (b) $\left(-\mu^{2} \leq E<0\right)$. In this case remember that $a<0$, and the reasoning is similar. Using (25), (26) and (27), we get

$$
\phi(x, y)=\cos (\sqrt{-E} y) C_{1}(x)+\sin (\sqrt{-E} y) C_{2}(x),
$$

where $C_{1}(x)=\frac{\mu}{a \alpha} J \phi_{x}(x, 0)$ and $C_{2}(x)=\frac{1}{\sqrt{-E}} \phi_{y}(x, 0)$. Again (25) and (28) imply that $C_{1}^{\prime}(x)=\frac{-i a \alpha}{\mu} C_{1}(x), C_{2}^{\prime}(x)=\frac{i \mu E}{a \alpha} C_{2}(x)$. Hence

$$
\phi(x, y)=\left(\frac{i \mu}{a \sqrt{\alpha}} \cos (\sqrt{-E} y) \exp \left(\frac{-i a \alpha}{\mu} x\right), \frac{\sqrt{\alpha}}{\sqrt{-E}} \sin (\sqrt{-E} y) \exp \left(\frac{i \mu E}{a \alpha} x\right)\right) .
$$

Introducing the new parameter $c:=g(0)>0$ and using (18), we also have $c=\frac{\mu^{2}}{\alpha a^{2}}$, and now (28) gives that $-1-2 a c=\frac{-\mu^{2} E}{\alpha^{2} a^{2}}$; observe that $-\mu^{2} \leq E<0$ only implies that $c>-1 /(2 a)$. Using the coordinates $x+i y=\frac{1}{\sqrt{-E}}(t+i s)$, we finally get

$$
\phi(t, s)=\left(-i \sqrt{c} \cos s \exp \left(\frac{i t}{\sqrt{-1-2 a c}}\right), \frac{\sqrt{c}}{\sqrt{-1-2 a c}} \sin s \exp (i \sqrt{-1-2 a c} t)\right),
$$

where $\frac{-1}{2 a}<c$. Finally we put $c=\frac{\cosh ^{2} \nu}{-2 a}, \nu>0$, and this proves part (b)(iv).

Corollary 1. The tori $\mathcal{T}_{m, n}$ (described in Proposition 3) are the only compact orientable Hamiltonian stationary self-similar solutions for mean curvature flow in the complex Euclidean plane.

\section{ACKNowledgments}

The authors would like to thank Francisco Urbano for his valuable suggestions and constant availability and Henri Anciaux for helpful discussions. 


\section{REFERENCES}

[1] H. Anciaux. Construction of Lagrangian self-similar solutions to the mean curvature flow in $\mathbb{C}^{n}$. Geom. Dedicata 120 (2006) 37-48. MR2252892 (2007h:53098)

[2] H. Anciaux and I. Castro. Constructions of Hamiltonian-minimal Lagrangian submanifolds in complex Euclidean space. Preprint, 2009. arXiv:0906.4305 [math.DG]

[3] I. Castro and B.-Y. Chen. Lagrangian surfaces in complex Euclidean plane via spherical and hyperbolic curves. Tohoku Math. J. (2) 58 (2006) 565-579. MR2297200 (2009h:53182)

[4] I. Castro and F. Urbano. Lagrangian surfaces in the complex Euclidean plane with conformal Maslov form. Tohoku Math. J. (2) 45 (1993) 565-582. MR.1245723 (94j:53064)

[5] I. Castro and F. Urbano. Examples of unstable Hamiltonian-minimal Lagrangian tori in $\mathbb{C}^{2}$. Compositio Math. 111 (1998) 1-14. MR 1611051 (98m:53075)

[6] J. Chen and J. Li. Singularity of mean curvature flow of Lagrangian submanifolds. Invent. Math. 156 (2004) 25-51. MR2047657 (2004m:53116)

[7] K. Groh, M. Schwarz, K. Smoczyk and K. Zehmisch. Mean curvature flow of monotone Lagrangian submanifolds. Math. Z. 257 (2007) 295-327. MR.2324804 (2008f:53090)

[8] R. Harvey and H.B. Lawson. Calibrated geometries. Acta Math. 148 (1982) 47-157. MR666108 (85i:53058)

[9] F. Hélein and P. Romon. Hamiltonian stationary Lagrangian surfaces in $\mathbb{C}^{2}$. Comm. Anal. Geom. 10 (2002) 79-126. MR.1894142 (2003e:53108)

[10] G. Huisken. Asymptotic behavior for singularities of the mean curvature flow. J. Differential Geom. 31 (1990) 285-299. MR.1030675 (90m:53016)

[11] D. Joyce, Y.-I. Lee and M.-P. Tsui. Self-similar solutions and translating solutions for Lagrangian mean curvature flow. arXiv:0801.3721 [math.DG]

[12] Y.-I. Lee and M.-T. Wang. Hamiltonian stationary shrinkers and expanders for Lagrangian mean curvature flows. in J. Differential Geom. 83 (2009), 27-42.

[13] Y.-I. Lee and M.-T. Wang. Hamiltonian stationary cones and self-similar solutions in higher dimension. Trans. Amer. Math. Soc. 362 (2010) 1491-1503.

[14] W.P. Minicozzi II. The Willmore functional on Lagrangian tori: Its relation to area and existence of smooth minimizers. J. Amer. Math. Soc. 8 (1995) 761-791. MR1311825|(95m:58038)

[15] A. Neves. Singularities of Lagrangian mean curvature flow: Zero-Maslov class case. Invent. Math. 168 (2007) 449-484. MR.2299559 (2008d:53092)

[16] A. Neves. Singularities of Lagrangian mean curvature flow: monotone case. arXiv:math 06068401v1 [math.DG], 2006.

[17] Y.G. Oh. Volume minimization of Lagrangian submanifolds under Hamiltonian deformations. Math. Z. 212 (1993) 175-192. MR.1202805 (94a:58040)

[18] K. Smoczyk. A canonical way to deform a Lagrangian submanifold. Preprint, dg-ga/9605005.

[19] R. Schoen and J.G. Wolfson. Minimizing area among Lagrangian surfaces: The mapping problem. J. Differential Geom. 57 (2001) 301-388. MR1895348 (2003c:53119)

[20] A. Strominger, S.-T. Yau and E. Zaslow. Mirror symmetry is T-duality. Nuclear Phys. B479 (1996) 243-259. MR1429831 (97j:32022)

[21] R.P. Thomas and S.-T. Yau. Special Lagrangians, stable bundles and mean curvature flow. Comm. Anal. Geom. 10 (2002) 1075-1113. MR.1957663 (2004c:53073)

[22] M.-T. Wang. Mean curvature flow of surfaces in Einstein four-manifolds. J. Differential Geom. 58 (2001) 1-86. MR.1879229(2003j:53108)

Departamento de Matemáticas, Universidad de JaÉn, 23071 Jaén, Spain

E-mail address: icastro@ujaen.es

Departamento de Matemáticas, Universidad de JaÉn, 23071 JaÉn, Spain

E-mail address: alerma@ujaen.es 